\title{
Arthroscopic Superior Capsular Reconstruction With Acellular Porcine Dermal Xenograft for the Treatment of Massive Irreparable Rotator Cuff Tears
}

\author{
Martin Polacek, M.D., Ph.D.
}

\begin{abstract}
Purpose: To evaluate the short-term clinical outcomes and the complications related to arthroscopic superior capsular reconstruction (SCR) with acellular porcine dermal xenograft for the treatment of irreparable massive rotator cuff tears. Methods: A prospective observational study of patients treated with arthroscopic SCR for irreparable massive rotator cuff tears in the period from 2016 to 2017 was performed. Range of motion and Shoulder Pain and Disability Index (SPADI) scores were assessed preoperatively, at 6 months postoperatively, and at 12 months postoperatively. Results: A total of 20 shoulders in 19 patients, with an average age of 60 years, were included in the study. Twelve patients met the criterion for the minimal clinically important difference in the SPADI score. The mean SPADI score showed significant improvement from $51.3 \%$ to $10.4 \%$ at 1 -year follow-up. Active abduction improved from $65.4^{\circ}$ to $149.3^{\circ}$ and active forward flexion improved from $68.6^{\circ}$ to $151.4^{\circ}$ at 1 -year follow-up. The procedure had a $30 \%$ complication rate, including a $15 \%$ rate of immunologic rejection of the xenograft. Five patients underwent revision procedures, including arthroscopic debridement and removal of xenograft residuals, implantation of a balloon spacer, and revision SCR with a fascia lata autograft. Conclusions: Arthroscopic SCR with an acellular porcine dermal xenograft led to a successful outcome in $60 \%$ of cases. The procedure showed a quite high complication rate; the most severe cases were related to acute immunologic rejection of the xenograft. Level of Evidence: Level IV, case series.
\end{abstract}

$\mathbf{T}$ he treatment of massive rotator cuff tears (MRCTs) is challenging. In some cases, MRCTs can be mobilized and repaired, but poor tissue quality and inelasticity of the tendon lead to a quite high failure rate. ${ }^{1}$ Chronic rotator cuff insufficiency might result in abnormal glenohumeral wear, superior migration of the humeral head, and glenohumeral cartilage destruction. ${ }^{2,3}$ The first line of treatment of irreparable MRCTs is usually conservative. Mainstays of nonoperative treatment include nonsteroidal anti-inflammatory drugs, subacromial corticosteroid injections, and

From the Orthopedic Department, Drammen Hospital, Vestre Viken Hospital Trust, Drammen, Norway.

The author reports the following potential conflicts of interest or sources of funding: M.P. receives support from Arthrex. Full ICMJE author disclosure forms are available for this article online, as supplementary material.

Received May 24, 2019; accepted August 17, 2019.

Address correspondence to Martin Polacek, M.D., Ph.D., Orthopedic Department, Drammen Hospital, Vestre Viken Hospital Trust, 3004 Drammen, Norway.E-mail:marpol@vestreviken.no

(C) 2019 by the Arthroscopy Association of North America. Published by Elsevier Inc. This is an open access article under the CC BY-NC-ND license (http://creativecommons.org/licenses/by-nc-nd/4.0/).

2666-061X/19651

https://doi.org/10.1016/j.asmr.2019.08.001 physiotherapy. Rehabilitation exercises focus on global deltoid reconditioning and periscapular strengthening and can help to restore the shoulder's functional range of motion (ROM). ${ }^{4}$ Nonsurgical treatment should be attempted for at least 6 months before surgery is considered. ${ }^{4-7}$ The most common surgical technique in the treatment of irreparable MRCTs is arthroscopic debridement. Early studies showed an approximately $85 \%$ success rate at short-term follow-up (minimum of 1 year), including improvement in ROM from less than $90^{\circ}$ to greater than $150^{\circ} .{ }^{8}$ However, in the long term, the results deteriorated. ${ }^{9}$ Other surgical techniques in the treatment of irreparable MRCTs include biceps release, biceps tenodesis, partial tendon repair, augmentation or bridge grafting of the rotator cuff tendon, and several tendon transfers. ${ }^{4,10-15}$ Lately, reverse shoulder arthroplasty (RSA) has emerged as a treatment option for MRCTs without arthropathy in patients younger than 60 years. ${ }^{16}$ RSA remains a successful and well-accepted option in a lower-demand patient population older than 70 years with chronic, irreparable MRCTs and rotator cuff arthropathy. ${ }^{17,18}$ However, the use of RSA in the management of MRCTs without arthritis in a more physically active patient population younger than 60 years remains 
controversial because of a relatively high complication rate of up to $17.4 \%$ and questionable long-term results. $^{18,19}$

Recently, the importance of the superior joint capsule in passive stability of the shoulder joint has been acknowledged. The superior joint capsule is located on the inferior surface of the supraspinatus and infraspinatus tendons. It has been reported that a tear in the superior joint capsule might lead to an abnormal load on the rotator cuff and significantly increase glenohumeral translation in all directions and subacromial contact pressure at $30^{\circ}$ of shoulder abduction. ${ }^{20,21}$

Mihata et al. ${ }^{20,22}$ introduced a technique, superior capsular reconstruction (SCR), in which the superior joint capsule was reconstructed by a fascia lata autograft. The graft was attached to the superior glenoid and the humeral head by suture anchors. ${ }^{20,22}$ The initial study by Mihata et al. ${ }^{22}$ showed excellent results with a high grade of patient satisfaction, significant improvement in shoulder function, and a low complication rate. Because of donor-site morbidity after the fascia lata harvest, the technique has been modified and an acellular dermal allograft has been introduced as an alternative. ${ }^{23,24}$

The purpose of this study was to evaluate the shortterm clinical outcomes and the complications related to arthroscopic SCR with acellular porcine dermal xenograft for the treatment of irreparable MRCTs. The hypothesis was that SCR with dermal xenograft would lead to significant clinical improvement in most patients with irreparable MRCTs at 1-year follow-up and the procedure would have a relatively low complication rate.

\section{Methods}

In the period from 2016 to 2017, a consecutive group of 19 patients ( 20 shoulders) were included in a prospective study and surgically treated with arthroscopic SCR using acellular porcine dermal xenograft. Written informed consent was obtained from the study participants, and the hospital's local ethical board approved the study. The patients were referred to our hospital by other surgeons, general practitioners, or physiotherapists based on a tentative diagnosis of an irreparable MRCT. They were then assessed by the treating surgeon at the hospital's outpatient clinic. Suitable patients were informed about the SCR procedure including its potential risks and the postoperative rehabilitation protocol. At the same time, they were invited to participate in our study. The inclusion criteria were based on clinical and radiologic findings. The study included patients with symptomatic, chronic MRCTs characterized by severe shoulder weakness, especially during active abduction and active forward flexion; pain; and impaired ROM. All patients had a symptom duration of at least 12 months and tried physiotherapy, with a focus on strengthening of the deltoid and periscapular muscles, for at least 6 months. Magnetic resonance imaging (MRI) scans were used to assess the rotator cuff tear with a focus on the grade of tendon retraction, fatty atrophy of the supraspinatus muscle, superior migration of the humeral head, and glenohumeral cartilage. The inclusion criteria based on the MRI findings were significant retraction of the supraspinatus tendon over 5 $\mathrm{cm}$ (Bateman classification grade 4), significant fatty atrophy of the supraspinatus muscle (Goutallier stage 4 ), light or no rotator cuff arthropathy (Hamada stages 1 and 2) without acetabularization, and intact or reparable subscapularis and infraspinatus tendons. ${ }^{25-27}$ The exclusion criteria included patients not motivated to follow the postoperative rehabilitation protocol and patients with known alcohol or drug abuse, a serious cardiovascular condition (American Society of Anesthesiologists class 3-5), or an acute systemic infection. Previous shoulder surgery was not an exclusion criterion.

Owing to the cost and availability of the hospital's MRI scans, postoperative imaging was conducted only in patients expressing dissatisfaction with postoperative outcomes, such as an increased pain level, shoulder weakness, and little or no functional improvement. SCR was performed by a single surgeon as an outpatient procedure in the hospital's day-surgery unit. The patients were assessed by the treating surgeon preoperatively, at 6 months postoperatively, and at 1 year postoperatively with the Shoulder Pain and Disability Index (SPADI). The SPADI questionnaire was administered preoperatively by a nurse in the day-surgery unit and postoperatively by the treating physiotherapist. ROM was assessed preoperatively and at 1 year postoperatively by the treating surgeon. Active abduction and active forward flexion were recorded. Any complications or revision surgical procedures were also recorded. On the basis of previous studies, the minimal clinically important difference in the SPADI score was $18 \%{ }^{28}$ Cases that required revision surgery were categorized as failures in this study.

\section{Surgical Technique}

The surgical technique has previously been described. ${ }^{20,23,24,29,30}$ The operation was performed with the patient in the lateral decubitus position with the arm in $30^{\circ}$ of abduction and $10^{\circ}$ of forward flexion. The portals commonly used for the procedure were as follows: posterior portal, posterolateral portal, port of Wilmington, lateral portal, anterosuperolateral portal, anterior portal, and Neviaser portal. First, the glenohumeral joint was assessed. The biceps tendon was released, and if necessary, the subscapularis tendon was repaired. The subacromial space was then cleaned. Although the critical shoulder angle was not measured preoperatively, an acromioplasty was performed in all 
patients. The supraspinatus and infraspinatus tendons were tested regarding their irreparability, and if necessary, the infraspinatus tendon was repaired. Partial repair of the supraspinatus was not attempted during the SCR procedure. In cases with an inadequate view, a posterior interval slide was performed to improve visualization of the superior glenoid. The superior glenoid was then prepared, with preservation of the superior labrum, and 2 or 3 anchors were placed in the superior glenoid. Two knotted anchors (Lupine; DePuy Synthes [Johnson \& Johnson], New Brunswick, $\mathrm{NJ}$ ) in 10- and 2-o'clock configurations were used in 8 cases, whereas 3 knotless anchors (Knotless SutureTak; Arthrex, Naples, FL) at the 10-, 12-, and 2-o'clock positions were used in 12 cases. The use of 2 knotted versus 3 knotless glenoid anchors might be explained by the development and improvement of the technical aspects of the procedure. Next, 2 anchors (SwiveLock, $4.75 \mathrm{~mm}$; Arthrex) preloaded with suture tape were placed in the greater tuberosity adjacent to the cartilage margin. The distance between the anchors was measured with a calibrated probe. A double DX Reinforcement Matrix graft (Arthrex) was then used. According to the manufacturer, the DX Reinforcement Matrix is a porcine acellular dermal extracellular matrix (ECM) that has been created by OPTRIX processing technology (DSM, Exton, PA) as a biomechanically strong and biocompatible scaffold for the reinforcement and repair of soft tissues. The thickness of the scaffold was $1.5 \mathrm{~mm}$. The graft size was extended $5 \mathrm{~mm}$ medially, $5 \mathrm{~mm}$ anteriorly, $5 \mathrm{~mm}$ posteriorly, and 10 mm laterally. The graft was sized, doubled, and secured with a suture. Sutures from each of the anchors, beginning with the anchors in the greater tuberosity, were retrieved out of the lateral portal and passed through the graft. The graft was then shuttled into the joint and secured to the superior glenoid. Next, the graft was secured to the greater tuberosity with a double-row configuration, using 2 anchors (SwiveLock, $4.75 \mathrm{~mm}$ ) in a SpeedBridge configuration (Arthrex). The arm was in $30^{\circ}$ of abduction and $10^{\circ}$ of forward flexion when the graft was secured. Finally, the graft was attached to the infraspinatus tendon with side-to-side sutures.
The postoperative rehabilitation protocol was as follows: The shoulder was immobilized in a sling for 6 weeks. Passive ROM was initiated after 2 weeks. After 6 weeks, passive and active-assisted ROM was allowed. Exercises strengthening the deltoid and periscapular muscles were allowed after 3 months. Return to full activity was allowed at 6 to 12 months.

\section{Statistical Analysis}

The SPADI score, as well as active abduction and forward flexion, of all treated patients are described as mean and standard deviation. The dependent $t$ test was used to analyze the preoperative and postoperative outcomes. $P<.05$ was considered statistically significant.

\section{Results}

A total of 20 treated shoulders in 19 consecutive patients (14 men and 5 women), with an average age of 60 years (range, 45-72 years), were included in the study. Follow-up data were obtained from all patients, and no patient was lost to follow-up. None of the patients included in the study with supraspinatus tendon retraction greater than $5 \mathrm{~cm}$ (Bateman classification grade 4), Goutallier stage 4 fatty atrophy of the supraspinatus muscle, and superior migration of the humeral head (Hamada stages 1 and 2) had a reparable supraspinatus tear. Three patients had undergone a previous arthroscopic rotator cuff repair. Additional procedures performed during SCR surgery included infraspinatus repair ( 1 case) and subscapularis repair (1 case). Biceps release and acromioplasty were performed in all cases.

Twelve patients met the criterion for the minimal clinically important difference in the SPADI score $(>18 \%)$ and experienced highly significant improvement in shoulder function. The mean SPADI score improved significantly at 1-year follow-up from $51.3 \%$ to $10.4 \%$ (Table 1 ). Figure 1 shows a gradual improvement in functional outcomes at 6 months and 1 year postoperatively.

Active ROM improved significantly as well. Specifically, mean active abduction improved from $65.4^{\circ}$ to $149.3^{\circ}$ and mean active forward flexion improved from $68.6^{\circ}$ to $151.4^{\circ}$ at 1 -year follow-up (Table 2 ).

Table 1. Functional Outcomes After Arthroscopic Superior Capsular Reconstruction

\begin{tabular}{lccc}
\hline & & SPADI Score, $\%$ \\
\cline { 2 - 4 } & \multicolumn{1}{c}{ Total } & Pain & Disability \\
\hline Preoperative & $51.3 \pm 19.2$ & $54.9 \pm 22.61$ & $49.2 \pm 21.4$ \\
6-mo follow-up & $23.1 \pm 18.1$ & $21.86 \pm 19.74$ & $23.6 \pm 18.7$ \\
12-mo follow-up & $10.4 \pm 8.8$ & $8.3 \pm 11.2$ & $11.1 \pm 8.8$ \\
Difference between & $40.9, P<.0001$ & $46.6, P<.0001$ & $38.1, P<.0001$ \\
$\quad$ preoperative and 12 mo & & & \\
\hline NOTE. Data are presented as mean \pm standard deviation or mean. & & \\
SPADI, Shoulder Pain and Disability Index.
\end{tabular}




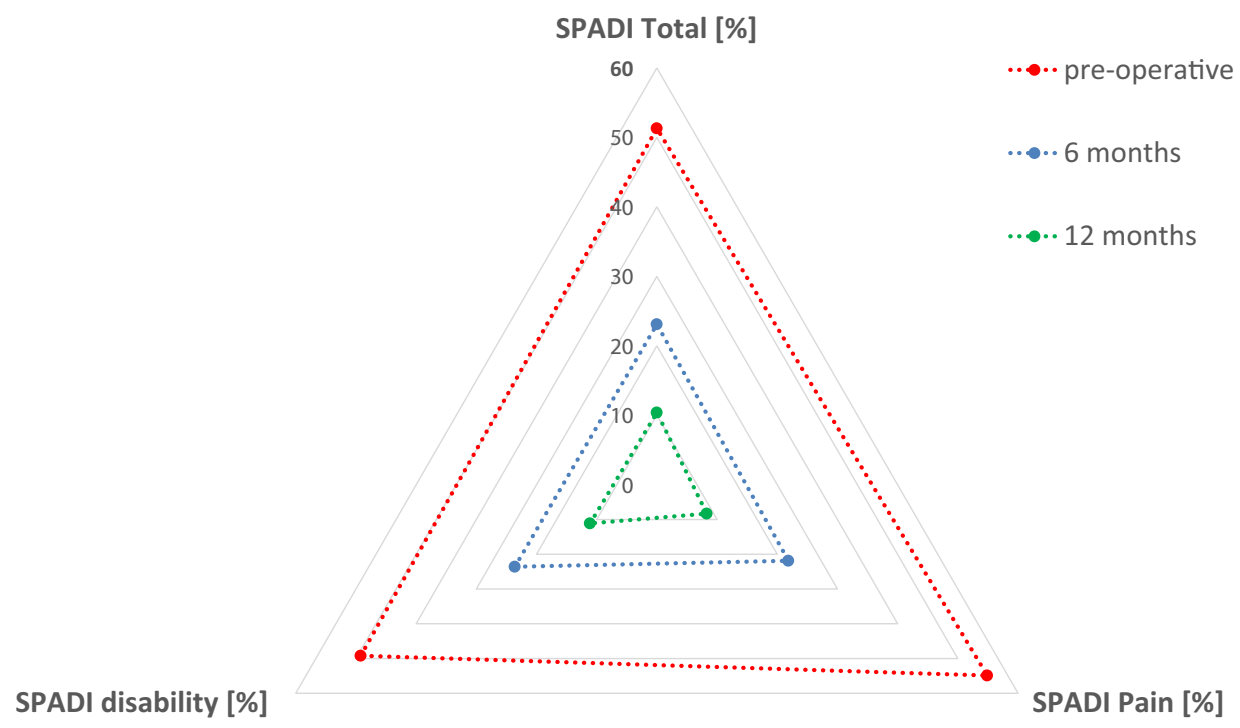

Fig 1. Shoulder function after superior capsular reconstruction with acellular porcine dermal xenograft assessed by the Shoulder Pain and Disability Index (SPADI). Patients were assessed with the SPADI questionnaire preoperatively, at 6 months postoperatively, and at 1 year postoperatively.

Postoperative complications were observed in 6 cases overall (Table 3). Postoperative acute immunologic rejection of the xenograft occurred in 3 of these cases. All of the affected patients presented with shoulder pain, swelling around the shoulder joint, and persistent secretion of clear fluid with small white particles from the lateral portal. In addition, systemic symptoms such as fatigue and fever were recorded. Initially, an acute bacterial joint infection was suspected. However, the blood test results showed only a slight increase in the Creactive protein level, a normal leukocyte count, an increased serum erythrocyte sedimentation rate, and an increased eosinophil count. Two patients had preexisting conditions that could alter the immune system: chronic low-grade osteomyelitis in the jaw and psoriatic arthritis. In the third patient, hospital-acquired pneumonia developed postoperatively. The patients were treated immediately with revision arthroscopy. Intraoperatively, it was discovered that the xenograft was completely degraded into small white particles around 2 to $3 \mathrm{~mm}$ in diameter. Graft debris and all suture material were removed. No wound-healing complications occurred after the revision surgical procedure. Approximately 3 weeks after the operation, the rehabilitation program was initiated with a focus on strengthening exercises. The patients were initially

Table 2. Range of Motion After Arthroscopic Superior Capsular Reconstruction

\begin{tabular}{lcc}
\hline & $\begin{array}{c}\text { Active } \\
\text { Abduction, }\end{array}$ & $\begin{array}{c}\text { Active Forward } \\
\text { Flexion, }{ }^{\circ}\end{array}$ \\
\hline Preoperative & $65.4 \pm 21.1$ & $68.6 \pm 21.4$ \\
12-mo follow-up & $149.3 \pm 28.7$ & $151.4 \pm 28.3$ \\
$\begin{array}{l}\text { Difference between } \\
\quad \text { preoperative and 12 mo }\end{array}$ & $83.9, P<.0001$ & $82.8, P<.0001$ \\
\hline
\end{tabular}

NOTE. Data are presented as mean \pm standard deviation or mean. treated with intravenous cloxacillin. However, cultures from the intraoperative samples did not show any bacterial growth, so antibiotic treatment was discontinued when the results of the cultures were negative. Two of the affected patients responded well to the rehabilitation program and did not require additional surgery. The third patient did not respond to the rehabilitation program; therefore, revision SCR using a fascia lata autograft was performed approximately 8 months after the initial operation. Postoperatively, the patient showed improvement in shoulder function and ROM (Table 3).

Three other patients were dissatisfied after the procedure and did not experience improvement in pain, weakness, and active ROM despite the postoperative rehabilitation program. In these cases, MRI examination revealed a rupture of the graft on the humeral head. A condition that might influence the healing potential was identified in 2 patients: One patient had juvenile rheumatoid arthritis, and the other patient was a smoker. The third patient did not have any preexisting conditions. However, progression of glenohumeral arthritis was discovered postoperatively. Two of these patients required additional surgery, but neither was interested in total shoulder replacement. Instead, they were treated with implantation of a subacromial balloon spacer (ISB; OrthoSpace, Caesarea, Israel).

\section{Discussion}

In this study, 12 of 20 cases met the criterion for the minimal clinically important difference in the SPADI score $(>18 \%)$. The patients experienced a significant improvement in the SPADI shoulder score by $40.9 \%$. Mean active abduction improved from $65.4^{\circ}$ to $149.3^{\circ}$ 


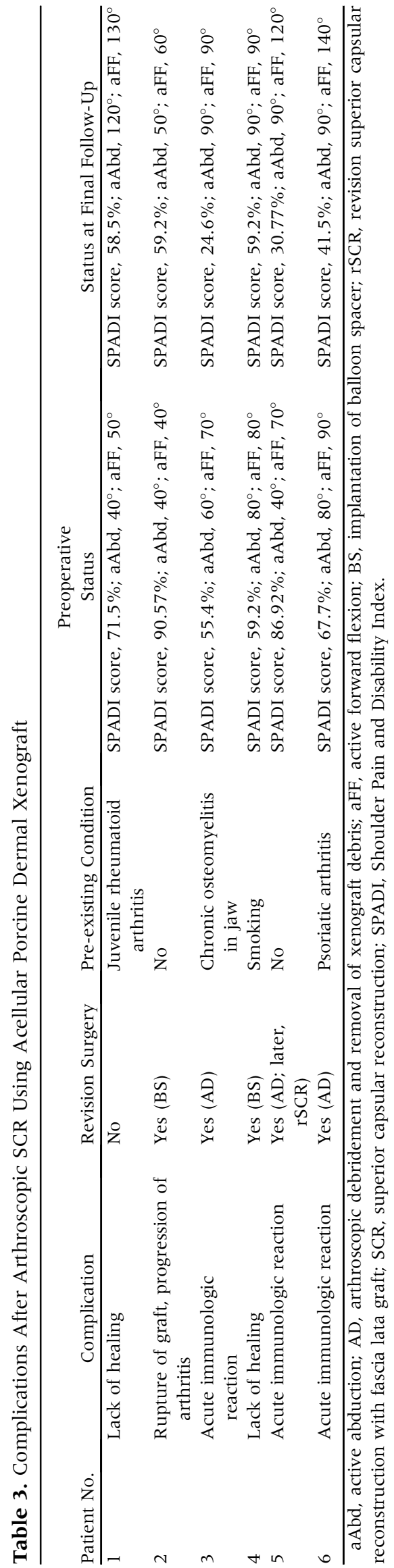

and mean active forward flexion improved from $68.6^{\circ}$ to $151.4^{\circ}$ at 1 -year follow-up. Our study showed a complication rate of $30 \%$, including a $15 \%$ rate of immunologic rejection of the xenograft. The onset of the immunologic reaction several weeks after implantation indicated acute immunologic rejection of the xenogeneic antigens. ${ }^{31}$ A specific condition that could alter the immunologic response was able to be identified in all cases. The first patient had chronic low-grade osteomyelitis in the jaw, the second had psoriatic arthritis, and the third acquired bacterial pneumonia after the surgical procedure. All of these conditions might lead to an alteration of the innate immune defense system that is in charge of responses to foreignbody antigens.

Biological scaffolds composed of xenogeneic or allogeneic ECMs have been widely used in both preclinical studies and clinical settings. ${ }^{32-35}$ The ECM scaffolds are usually prepared by decellularization of the tissue and removal of the immunogenic antigens. ${ }^{36} \mathrm{~A}$ tremendous amount of research has been performed to develop allografts and xenografts with a low risk of immunologic host reactions, high host cell ingrowth, ECM turnover, and incorporation of the graft into the host tissue. However, residual antigenicity of the biological scaffolds remains an issue. ${ }^{36,37}$ The graft preparation process has improved substantially because of an increased understanding of the immunogenic antigens in xenogeneic tissues and mechanisms of immunologic host reactions. ${ }^{31,36}$ It has been shown that extensive decellularization of the native tissues leads to disruption of the ECM and poor mechanical properties of the graft. In addition, preservation of the native ECM architecture such as tissue morphology, alignment of the collagen fibers, and thermal stability is critical in avoiding the innate immune system-mediated foreignbody response toward the ECM scaffold and facilitating recellularization of the scaffold. ${ }^{31}$ Preservation of the ECM during the preparation process is usually achieved by stepwise solubilization of both the hydrophilic and lipophilic antigens in the ECM in addition to the cell and DNA removal. ${ }^{31,33,38-40}$ However, total removal of the antigens from xenogeneic or allogeneic tissues is not an attainable goal to maintain the structural properties and the recellularization potential. Studies have also shown that total removal of the antigens is not a necessary criterion for a functioning xenograft. ${ }^{31}$ ECM turnover begins around 1 month after implantation of the scaffold, and complete ECM turnover occurs approximately 6 months after implantation. ${ }^{41}$ Therefore, if the tissue antigenicity can be sufficiently reduced to avoid immune-mediated destruction for approximately 6 months, the original xenogeneic scaffold is completely replaced by the recipient ECM. ${ }^{31,41} \mathrm{~A}$ study using a primate model showed increasing integration of the implanted porcine graft at 3 months after 
implantation. ${ }^{42}$ At 6 months, it was difficult to distinguish the graft from the native recipient tissue.

The $\alpha$-gal epitopes are the main antigenic factor in a xenograft. The long-term anti-non-gal antibody response has been studied in patients who underwent implantation of a porcine xenogeneic ligament replacing the torn anterior cruciate ligament. ${ }^{43,44}$ The implanted tendon was gradually replaced by host collagen fibers without impairing the biomechanical properties of the graft. The recipients displayed no antibody response in the first 3 weeks after implantation. However, the anti-non-gal antibodies appeared in all patients after 2 months and peaked by 6 months after implantation. Antibody activity decreased at 1 year and disappeared at 2 years. ${ }^{44}$ These observations suggest that the recipient's immune system was stimulated to produce antibodies as long as the porcine tissue was present in the body. The immunologic response did not affect the mechanical properties of the implanted material, and by 24 months, all of the porcine tissue was replaced by human ligament tissue. The xenografts and surgery were well tolerated by all patients. At 24 months' follow-up, 5 of 6 evaluated subjects presented with functional xenografts that were clinically satisfactory and lacked knee effusion, which if present would reflect extensive inflammation. One subject presented with tibial bone plug loosening at 15 months after implantation. No cases of immunologic rejection of the xenograft have been reported. ${ }^{44}$

Another study reported on the use of interposition acellular porcine dermal xenograft in patients with MRCTs. ${ }^{45}$ The study included 60 patients $(61$ shoulders) and reported significant improvements in pain, ROM, and manual muscle strength. Specifically, the mean visual analog scale (VAS) score decreased from 4.0 preoperatively to 1.0 postoperatively. Mean active forward flexion improved from $140.7^{\circ}$ to $160.4^{\circ}$, external rotation at $0^{\circ}$ of abduction improved from $55.6^{\circ}$ to $70.1^{\circ}$, and internal rotation at $90^{\circ}$ of abduction improved from $52.0^{\circ}$ to $76.2^{\circ}$. The average modified American Shoulder and Elbow Surgeons (ASES) score was 87.8 postoperatively. Musculoskeletal ultrasound showed that $91.8 \%$ of repairs (56 of 61) were fully intact; $3.3 \%$ ( 2 of 61 ), partially intact; and $4.9 \%$ ( 3 of $61)$, not intact. No infections, evidence of inflammatory reaction, tissue rejection, or other major adverse outcomes were reported. ${ }^{45}$

Other experiments in different animal models such as pigs, primates, and rabbits also showed that a mild immune response does not seem to have a negative impact on the regenerative potential of the implanted material. ${ }^{31,46}$ In comparison with these studies, the rejection rate in our study was quite high. In retrospect, with acknowledgment of the immunologic reaction pattern of the host organism to the xenografts, the patients with pre-existing conditions altering the immune defense system, such as chronic osteomyelitis, juvenile rheumatoid arthritis, and psoriatic arthritis, should not have been treated with the xenograft and hence should not have been included in the study.

The grafts commonly used in SCR are either an acellular dermal allograft or fascia lata autograft. Mihata et al. ${ }^{22}$ reported the outcomes of 24 shoulders in 23 patients treated with SCR using a fascia lata autograft. The patients showed significant improvement in shoulder function. Specifically, the ASES score improved from 23.5 to 92.9 , mean active elevation increased from $84^{\circ}$ to $148^{\circ}$, and external rotation increased from $26^{\circ}$ to $40^{\circ}$. The acromiohumeral distance increased from 4.6 to $8.7 \mathrm{~mm}$. Postoperative MRI scans showed an $83.3 \%$ healing rate of the implanted fascia lata autograft and repaired rotator cuff tendons. Three patients experienced retears of the repaired infraspinatus tendons, and 1 had a tear of the autograft. No cases of arthritis progression or rotator cuff muscle atrophy occurred. No complications such as neural injury, infection, or suture anchor problems were reported. In a more recent study, Mihata et al. ${ }^{47}$ reported the outcomes of 100 patients treated with SCR using a fascia lata autograft. The patients showed improvement in the ASES score from 36 to 92, active elevation improved by $56^{\circ}$, and external rotation improved by $15^{\circ}$. A $16 \%$ complication rate was noted, comprising 2 deep infections, 4 cases of suture anchor pullout, 2 severe joint contractures, 3 cases of fatty atrophy of the infraspinatus muscle, and 5 postoperative graft tears. Double or triple fascia lata autograft with a final thickness of 6 to $8 \mathrm{~mm}$ was used in both studies. Despite a quite large harvesting area (approximately $5 \times 12$ $\mathrm{cm})$, none of the patients reported donor-site morbidity or dysfunction.

Denard et al. ${ }^{25}$ reported the outcomes of 59 patients treated with an SCR procedure using an acellular dermal allograft. Active forward flexion improved from $130^{\circ}$ to $158^{\circ}$, and external rotation improved from $26^{\circ}$ to $45^{\circ}$. The VAS score decreased from 5.8 to 1.7 , the ASES score improved from 43.6 to 77.5 , and the Subjective Shoulder Value (SSV) improved from 35.0 to 76.3. The graft thickness was $1 \mathrm{~mm}$ in 5 cases, $2 \mathrm{~mm}$ in 2 cases, and $3 \mathrm{~mm}$ in all the other cases. Postoperative MRI scans showed healing of the graft in $45 \%$ of patients, whereas the 1-mm grafts had a failure rate of up to $60 \%$. Overall, a $67.8 \%$ success rate was reported. Revision surgical procedures included RSA in 7 cases and revision SCR in 2. Complications included infection in 1 case and persistent pain after the biceps tenodesis in 1 patient.

Pennington et al. ${ }^{30}$ reported the outcomes of 86 patients treated with SCR using dermal allograft. The patients showed improvement in the VAS score from 4.0 to 1.5 and the ASES score from 52 to 82 at 1 -year follow-up. Active abduction increased from $103^{\circ}$ to 
$159^{\circ}$, and active forward flexion increased from $121^{\circ}$ to $160^{\circ}$. All patients received 3-mm-thick dermal allografts. Only a few complications and a failure rate of $4.5 \%$ were reported. Three graft tears were revealed by MRI scans, and 1 patient experienced increased pain and lack of function. Revision surgery was required in 1 patient, who underwent RSA. Postoperative MRI scans were conducted only in patients reporting dissatisfaction; thus, no graft healing rate was reported.

The improvements in the shoulder scores and ROM in the aforementioned studies followed a similar pattern to that in our study. Although Pennington et al. ${ }^{30}$ reported a very low complication rate, the fascia lata autograft seems to have superior clinical results and a superior healing rate to both allografts and xenografts. SCR procedures with a dermal xenograft seem to have a high rate of graft-related complications, specifically immunologic rejection of the graft. None of the authors using allograft reported on immunologic rejection of the graft after surgery.

The thickness of the graft may be important in achieving good clinical outcomes after the procedure. Our study used a 3-mm-thick xenograft. Allografts used in SCR procedures are usually $3 \mathrm{~mm}$ thick, and the fascia lata graft is usually 6 to $8 \mathrm{~mm}$ thick. Mihata et al. ${ }^{48}$ compared a 4-mm-thick fascia lata graft with an 8 -mm-thick fascia lata graft. Biomechanical analysis showed that although both the 4- and 8-mm-thick grafts decreased subacromial contact pressure, the 8mm-thick graft was better in reducing superior translation. Another study compared the biomechanical properties of a 3-mm-thick dermal allograft versus an 8-mm-thick fascia lata autograft in an SCR cadaveric model. ${ }^{49}$ The allografts were significantly elongated by $15 \%$ during testing, whereas the fascia lata graft lengths were unchanged. The dermal allografts partially restored superior glenohumeral stability, whereas the fascia lata grafts completely restored superior glenohumeral stability.

Postoperative MRI scans of all the patients treated with the SCR procedure could not be obtained in our study. Hence, the xenograft healing rate cannot be reported. However, with acknowledgment of our complication rate of $30 \%$ and revision rate of $25 \%$, both mostly graft related, our healing rate is probably lower than the reported healing rate of fascia lata autografts.

Total RSA has recently emerged as an option in the treatment of irreparable MRCTs. ${ }^{16,17,19}$ Ernstbrunner et al. ${ }^{16}$ reported on long-term outcomes after RSA for irreparable MRCTs in 20 patients younger than 60 years. The Constant score improved from 24 to 59 , and the SSV increased from $20 \%$ to $71 \%$. In addition, significant improvements were noted in active anterior elevation (from $64^{\circ}$ to $117^{\circ}$ ), active abduction (from $58^{\circ}$ to $111^{\circ}$ ), pain scores, and strength. The clinical outcomes did not significantly deteriorate beyond 10 years, and the functional results of patients with previous surgical procedures were not significantly inferior to those of patients with primary RSA. The complication rate was 39\%; complications included persistent stiffness, pain, mechanical block, early dislocation, late dislocation, glenoid component dissociation, and infection. The revision rate was 26\%; the revision procedures comprised arthroscopic debridement in 2 patients, exchange of the liner in 4 patients, conversion to hemiarthroplasty in 1 patient, and surgery for infection in 2 patients.

Petrillo et al. ${ }^{17}$ reported on outcomes and complications of RSA in 396 patients with an average age of 71.9 years. After surgery, all clinical scores improved in a statistically significant fashion. Specifically, the VAS score improved from 6.5 to 1.8 , the ASES score improved from 29.4 to 72.2 , the Constant shoulder score improved from 31.4 to 60.3 , and the SPADI score improved from $77 \%$ to $34 \%$. In addition, active abduction improved from $41.1^{\circ}$ to $115.4^{\circ}$, and active forward flexion improved from $51^{\circ}$ to $124.4^{\circ}$. The complication rate was $17.4 \%$. Complications included infection; humeral, scapular, and/or acromial fracture; humeral stem loosening; baseplate failure; center screw breakage; luxation; and mechanical failure. The rate of revision surgery was $7.3 \%$; revision procedures included change of the liner, revision of the humeral stem or glenoid plate, and revision owing to infection. An earlier study of RSA reported a complication rate of nearly $50 \%$ with a revision rate of $30 \%{ }^{50}$ Nevertheless, more recent studies have shown a dramatic decrease in the complication rate. ${ }^{51,52}$ If compared with our results, whether using autograft, allograft, or xenograft, the clinical improvement after RSA is quite similar. However, there are several important differences. The patient population treated with RSA in the study of Petrillo et al. was older (mean age, 71.9 years) than our patient population (mean age, 60 years) at the time of SCR surgery. RSA in patients younger than 60 years showed a much higher complication rate than in older individuals (aged 71.9 years). Although our study shows a quite high complication and revision rate in the context of SCR surgery, the rate is similar to that of RSA. On the other hand, although most of our complications are relatively easily solved by revision arthroscopy, the complications after RSA procedures are more severe and difficult to address.

Other procedures traditionally used for irreparable, chronic MRCTs are different tendon transfers. Specifically, latissimus dorsi transfer is used in patients with chronic posterosuperior cuff insufficiency, and pectoralis major transfer is used in patients with chronic anterosuperior rotator cuff insufficiency. ${ }^{4,53-56}$ Gerber et al. $^{57}$ reported the 10 -year results of 44 patients treated with latissimus dorsi transfer for chronic 
MRCTs with posterosuperior rotator cuff deficiency. At the final follow-up, the SSV improved from $29 \%$ to $70 \%$, the relative Constant score improved from $56 \%$ to $80 \%$, active abduction improved from $112.1^{\circ}$ to $122.6^{\circ}$, and active forward flexion improved from $118^{\circ}$ to $132.4^{\circ}$. Complications included 1 infection, 2 cases of postoperative shoulder stiffness, 1 latissimus dorsi avulsion, 1 deltoid avulsion, and 2 cases of ulnar nerve dysesthesia. The revision rate was $12.3 \%$; revision procedures included arthroscopic debridement, latissimus dorsi reconstruction, and RSA. El-Azab et al. $^{53}$ reported the results of 108 patients treated with latissimus dorsi transfer for chronic posterosuperior rotator cuff insufficiency with an average of 9.3 years' follow-up. At the final follow-up, the relative Constant score improved from $44 \%$ to $71 \%$, the ASES score improved from 30 to 70 , the VAS score improved from 7.8 to 2.4 , active abduction improved from $88.7^{\circ}$ to $127.4^{\circ}$, and forward flexion improved from $86^{\circ}$ to $133.5^{\circ}$. Complications included axillary hematoma in 4 cases, a radial nerve lesion in 1 , an axillary nerve lesion in 1 , deltoid insufficiency in 3, and secondary frozen shoulder in 2. Except for a hematoma evacuation, no revision surgical procedures were reported. By comparison, our study showed similar improvements in shoulder function and ROM. Although our complication rate was higher, the complications were relatively easily solved by revision arthroscopy. In addition, tendon transfer is a much more extensive and invasive surgical procedure than SCR.

\section{Limitations}

Our study has limitations. First, our initial experience is presented with a relatively low number of patients and without a control group. Inaccurate exclusion criteria, especially regarding pre-existing conditions that could impair healing of the xenograft or lead to an immunologic rejection, might have influenced the results and the complication rate. Second, only shortterm results are presented, and the surgical outcomes in the long term are not known. Third, postoperative MRI scans were not available to confirm healing of the graft in patients with significant improvements and successful outcomes after SCR surgery.

\section{Conclusions}

Arthroscopic SCR with an acellular porcine dermal xenograft led to a successful outcome in $60 \%$ of cases. The procedure showed a quite high complication rate; the most severe cases were related to acute immunologic rejection of the xenograft.

\section{References}

1. Le BT, Wu XL, Lam PH, Murrell GA. Factors predicting rotator cuff retears: An analysis of 1000 consecutive rotator cuff repairs. Am J Sports Med 2014;42:1134-1142.
2. Galatz LM, Ball CM, Teefey SA, Middleton WD, Yamaguchi K. The outcome and repair integrity of completely arthroscopically repaired large and massive rotator cuff tears. J Bone Joint Surg Am 2004;86:219-224.

3. Paxton ES, Teefey SA, Dahiya N, Keener JD, Yamaguchi K, Galatz LM. Clinical and radiographic outcomes of failed repairs of large or massive rotator cuff tears: Minimum ten-year follow-up. J Bone Joint Surg Am 2013;95:627-632.

4. Ladermann A, Collin P, Athwal GS, Scheibel M, Zumstein MA, Nourissat G. Current concepts in the primary management of irreparable posterosuperior rotator cuff tears without arthritis. EFORT Open Rev 2018;3: 200-209.

5. Ainsworth R. Physiotherapy rehabilitation in patients with massive, irreparable rotator cuff tears. Musculoskeletal Care 2006;4:140-151.

6. Levy O, Mullett H, Roberts S, Copeland S. The role of anterior deltoid reeducation in patients with massive irreparable degenerative rotator cuff tears. J Shoulder Elbow Surg 2008; 17:863-870.

7. Yian EH, Sodl JF, Dionysian E, Schneeberger AG. Anterior deltoid reeducation for irreparable rotator cuff tears revisited. J Shoulder Elbow Surg 2017;26:1562-1565.

8. Levy HJ, Gardner RD, Lemak LJ. Arthroscopic subacromial decompression in the treatment of full-thickness rotator cuff tears. Arthroscopy 1991;7:8-13.

9. Zvijac JE, Levy HJ, Lemak LJ. Arthroscopic subacromial decompression in the treatment of full thickness rotator cuff tears: A 3- to 6-year follow-up. Arthroscopy 1994;10: 518-523.

10. Barber FA, Burns JP, Deutsch A, Labbe MR, Litchfield RB. A prospective, randomized evaluation of acellular human dermal matrix augmentation for arthroscopic rotator cuff repair. Arthroscopy 2012;28:8-15.

11. Boileau P, Baque F, Valerio L, Ahrens P, Chuinard C, Trojani C. Isolated arthroscopic biceps tenotomy or tenodesis improves symptoms in patients with massive irreparable rotator cuff tears. J Bone Joint Surg Am 2007;89:747-757.

12. Bond JL, Dopirak RM, Higgins J, Burns J, Snyder SJ. Arthroscopic replacement of massive, irreparable rotator cuff tears using a GraftJacket allograft: Technique and preliminary results. Arthroscopy 2008;24:403-409.el.

13. Henseler JF, Nagels J, van der Zwaal P, Nelissen RG. Teres major tendon transfer for patients with massive irreparable posterosuperior rotator cuff tears: Short-term clinical results. Bone Joint J 2013;95-B:523-529.

14. Jones CR, Snyder SJ. Massive irreparable rotator cuff tears: A solution that bridges the gap. Sports Med Arthrosc Rev 2015;23:130-138.

15. Kanatli U, Ozer M, Ataoglu MB, et al. Arthroscopicassisted latissimus dorsi tendon transfer for massive, irreparable rotator cuff tears: Technique and short-term follow-up of patients with pseudoparalysis. Arthroscopy 2017;33:929-937.

16. Ernstbrunner L, Suter A, Catanzaro S, Rahm S, Gerber C. Reverse total shoulder arthroplasty for massive, irreparable rotator cuff tears before the age of 60 years: Long-term results. J Bone Joint Surg Am 2017;99:1721-1729. 
17. Petrillo S, Longo UG, Papalia R, Denaro V. Reverse shoulder arthroplasty for massive irreparable rotator cuff tears and cuff tear arthropathy: A systematic review. Musculoskelet Surg 2017;101:105-112.

18. Sellers TR, Abdelfattah A, Frankle MA. Massive rotator cuff tear: When to consider reverse shoulder arthroplasty. Curr Rev Musculoskelet Med 2018;11:131-140.

19. Sershon RA, Van Thiel GS, Lin EC, et al. Clinical outcomes of reverse total shoulder arthroplasty in patients aged younger than 60 years. J Shoulder Elbow Surg 2014;23: 395-400.

20. Mihata T, McGarry MH, Pirolo JM, Kinoshita M, Lee TQ. Superior capsule reconstruction to restore superior stability in irreparable rotator cuff tears: A biomechanical cadaveric study. Am J Sports Med 2012;40:2248-2255.

21. Ishihara Y, Mihata T, Tamboli M, et al. Role of the superior shoulder capsule in passive stability of the glenohumeral joint. J Shoulder Elbow Surg 2014;23:642-648.

22. Mihata T, Lee TQ, Watanabe C, et al. Clinical results of arthroscopic superior capsule reconstruction for irreparable rotator cuff tears. Arthroscopy 2013;29:459-470.

23. Adams CR, Denard PJ, Brady PC, Hartzler RU, Burkhart SS. The arthroscopic superior capsular reconstruction. Am J Orthop (Belle Mead NJ) 2016;45:320-324.

24. Burkhart SS, Denard PJ, Adams CR, Brady PC, Hartzler RU. Arthroscopic superior capsular reconstruction for massive irreparable rotator cuff repair. Arthrosc Tech 2016;5:e1407-e1418.

25. Denard PJ, Brady PC, Adams CR, Tokish JM, Burkhart SS. Preliminary results of arthroscopic superior capsule reconstruction with dermal allograft. Arthroscopy 2018;34:93-99.

26. Liotard JP, Cochard P, Walch G. Critical analysis of the supraspinatus outlet view: Rationale for a standard scapular Y-view. J Shoulder Elbow Surg 1998;7:134-139.

27. Nove-Josserand L, Edwards TB, O'Connor DP, Walch G. The acromiohumeral and coracohumeral intervals are abnormal in rotator cuff tears with muscular fatty degeneration. Clin Orthop Relat Res 2005:90-96.

28. Roy JS, MacDermid JC, Woodhouse LJ. Measuring shoulder function: A systematic review of four questionnaires. Arthritis Rheum 2009;61:623-632.

29. Hartzler RU, Burkhart SS. Superior capsular reconstruction. Orthopedics 2017;40:271-280.

30. Pennington WT, Bartz BA, Pauli JM, Walker CE, Schmidt W. Arthroscopic superior capsular reconstruction with acellular dermal allograft for the treatment of massive irreparable rotator cuff tears: Short-term clinical outcomes and the radiographic parameter of superior capsular distance. Arthroscopy 2018;34:1764-1773.

31. Wong ML, Wong JL, Vapniarsky N, Griffiths LG. In vivo xenogeneic scaffold fate is determined by residual antigenicity and extracellular matrix preservation. Biomaterials 2016;92:1-12.

32. Ansaloni L, Cambrini $\mathrm{P}$, Catena F, et al. Immune response to small intestinal submucosa (surgisis) implant in humans: Preliminary observations. J Invest Surg 2007;20: 237-241.

33. Hoganson DM, O'Doherty EM, Owens GE, et al. The retention of extracellular matrix proteins and angiogenic and mitogenic cytokines in a decellularized porcine dermis. Biomaterials 2010;31:6730-6737.

34. Mantovani F, Trinchieri A, Castelnuovo C, Romano AL, Pisani E. Reconstructive urethroplasty using porcine acellular matrix. Eur Urol 2003;44:600-602.

35. Smith M, Hooks VH, Jenkins B. Patch repair of ileoanal pouch-vaginal fistula with Permacol collagen implant. Am Surg 2007;73:514-515.

36. Wong ML, Griffiths LG. Immunogenicity in xenogeneic scaffold generation: Antigen removal vs. decellularization. Acta Biomater 2014;10:1806-1816.

37. Goncalves AC, Griffiths LG, Anthony RV, Orton EC. Decellularization of bovine pericardium for tissueengineering by targeted removal of xenoantigens. J Heart Valve Dis 2005;14:212-217.

38. Cissell DD, Hu JC, Griffiths LG, Athanasiou KA. Antigen removal for the production of biomechanically functional, xenogeneic tissue grafts. J Biomech 2014;47:1987-1996.

39. Keane TJ, Badylak SF. The host response to allogeneic and xenogeneic biological scaffold materials. J Tissue Eng Regen Med 2015;9:504-511.

40. Keane TJ, Swinehart IT, Badylak SF. Methods of tissue decellularization used for preparation of biologic scaffolds and in vivo relevance. Methods 2015;84:25-34.

41. Bayrak A, Tyralla M, Ladhoff J, et al. Human immune responses to porcine xenogeneic matrices and their extracellular matrix constituents in vitro. Biomaterials 2010;31:3793-3803.

42. Xu H, Sandor M, Qi S, et al. Implantation of a porcine acellular dermal graft in a primate model of rotator cuff repair. J Shoulder Elbow Surg 2012;21:580-588.

43. Galili U. Induced anti-non gal antibodies in human xenograft recipients. Transplantation 2012;93:1 1-16.

44. Stone KR, Abdel-Motal UM, Walgenbach AW, Turek TJ, Galili U. Replacement of human anterior cruciate ligaments with pig ligaments: A model for anti-non-gal antibody response in long-term xenotransplantation. Transplantation 2007;83:211-219.

45. Neumann JA, Zgonis MH, Rickert KD, et al. Interposition dermal matrix xenografts: A successful alternative to traditional treatment of massive rotator cuff tears. Am J Sports Med 2017;45:1261-1268.

46. Stone KR, Walgenbach AW, Turek TJ, Somers DL, Wicomb W, Galili U. Anterior cruciate ligament reconstruction with a porcine xenograft: A serologic, histologic, and biomechanical study in primates. Arthroscopy 2007;23: 411-419.

47. Mihata T, Lee TQ, Fukunishi K, et al. Return to sports and physical work after arthroscopic superior capsule reconstruction among patients with irreparable rotator cuff tears. Am J Sports Med 2018;46:1077-1083.

48. Mihata T, McGarry MH, Kahn T, Goldberg I, Neo M, Lee TQ. Biomechanical effect of thickness and tension of fascia lata graft on glenohumeral stability for superior capsule reconstruction in irreparable supraspinatus tears. Arthroscopy 2016;32:418-426.

49. Mihata T, Bui CNH, Akeda M, et al. A biomechanical cadaveric study comparing superior capsule reconstruction using fascia lata allograft with human dermal 
allograft for irreparable rotator cuff tear. J Shoulder Elbow Surg 2017;26:2158-2166.

50. Werner CM, Steinmann PA, Gilbart M, Gerber C. Treatment of painful pseudoparesis due to irreparable rotator cuff dysfunction with the Delta III reverse-ball-and-socket total shoulder prosthesis. J Bone Joint Surg Am 2005;87: 1476-1486.

51. Bohsali KI, Bois AJ, Wirth MA. Complications of shoulder arthroplasty. J Bone Joint Surg Am 2017;99:256-269.

52. Groh GI, Groh GM. Complications rates, reoperation rates, and the learning curve in reverse shoulder arthroplasty. J Shoulder Elbow Surg 2014;23:388-394.

53. El-Azab HM, Rott O, Irlenbusch U. Long-term follow-up after latissimus dorsi transfer for irreparable posterosuperior rotator cuff tears. J Bone Joint Surg Am 2015;97: 462-469.
54. Gerber C. Latissimus dorsi transfer for the treatment of irreparable tears of the rotator cuff. Clin Orthop Relat Res 1992:152-160.

55. Grimberg J, Kany J, Valenti P, Amaravathi R, Ramalingam AT. Arthroscopic-assisted latissimus dorsi tendon transfer for irreparable posterosuperior cuff tears. Arthroscopy 2015;31:599-607.el.

56. Moroder P, Schulz E, Mitterer M, Plachel F, Resch H, Lederer S. Long-term outcome after pectoralis major transfer for irreparable anterosuperior rotator cuff tears. J Bone Joint Surg Am 2017;99:239-245.

57. Gerber C, Rahm SA, Catanzaro S, Farshad M, Moor BK. Latissimus dorsi tendon transfer for treatment of irreparable posterosuperior rotator cuff tears: Long-term results at a minimum follow-up of ten years. J Bone Joint Surg Am 2013;95:1920-1926. 